Schneeweiß, Shalabh:

On the Estimation of the Linear Relation when the Error Variances are known

Sonderforschungsbereich 386, Paper 493 (2006)

Online unter: http://epub.ub.uni-muenchen.de/

Projektpartner
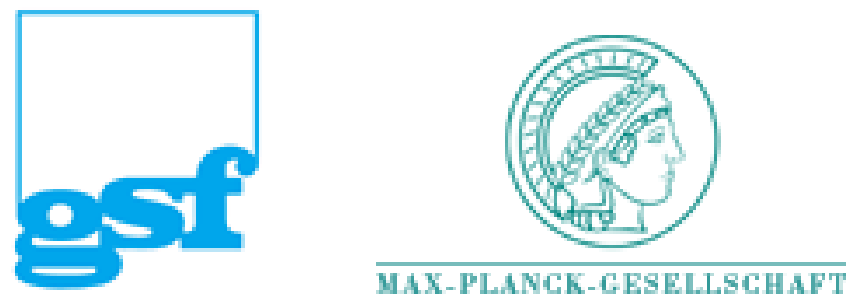


\title{
On the Estimation of the Linear Relation when the Error Variances are known
}

\author{
H. Schneeweiss \\ Department of Statistics \\ University of Munich \\ 80799 Munich (Germany) \\ E-mail: Hans.Schneeweiss@stat.uni-muenchen.de \\ Shalabh \\ Department of Mathematics and Statistics \\ Indian Institute of Technology \\ Kanpur-208016 (India) \\ E-mail: shalab@iitk.ac.in; shalabh1@yahoo.com \\ Discussion Paper 493 SFB386 \\ University of Munich, Munich
}

\begin{abstract}
The present article considers the problem of consistent estimation in measurement error models. A linear relation with not necessarily normally distributed measurement errors is considered. Three possible estimators which are constructed as different combinations of the estimators arising from direct and inverse regression are considered. The efficiency properties of these three estimators are derived and analyzed. The effect of non-normally distributed measurement errors is analyzed. A Monte-Carlo experiment is conducted to study the performance of these estimators in finite samples and the effect of a non-normal distribution of the measurement errors.
\end{abstract}




\section{Introduction:}

In a linear measurement error model, the parameters can be estimated consistently only when some additional information besides the data set is available. There are various ways such additional information can be employed; e.g.; Cheng and Van Ness (1999) and Fuller (1987). Among them, application of the knowledge of all or one of the measurement error variances is the most prominent approach.

There are three basic consistent estimators of the slope parameter of the linear model depending on what knowledge is used. If only one of the two error variances is known and used, estimators can be constructed by adjusting either the direct (or ordinary) least squares (LS) or the inverse least squares estimator for measurement errors. A third, most prominent, estimator is orthogonal (or total) least squares (TLS), which relies on the knowledge of the ratio of the error variances. For normally distributed measurement errors, this latter estimator is the maximum likelihood estimator. However the former two can be combined in various ways to construct new estimators that can compete with the TLS estimator, in particular in small samples and when the measurement errors are non-normal.

We have considered three such combinations of the direct and inverse adjusted LS estimators. They are modelled after analogous combinations found in the literature, where, however, they have been constructed from non-adjusted direct and inverse LS estimators. Sokal and Rohalf (1981) considered the geometric mean of these two estimators (which they call the technique of reduced major axis) and Aaronson et al. (1986) work with the arithmetic mean. In addition, the slope parameter may be estimated by the slope of the line that bisects the angle between the direct and inverse regression lines; see, e.g., Pierce and Tully (1988). While all these estimators are not consistent (although they possibly reduce the bias inherent in their constituent direct and inverse LS estimators), the present paper constructs consistent estimators by using error adjusted direct and inverse LS rather then non-adjusted direct and inverse LS estimators. A 
simple question then arises: which out of these suggested estimators is better under what conditions. This question has been partly dealt with in Dorff and Gurland (1969), but for a model with replicated observations and unknown error variances.

It seems plausible that the reliability ratios associated with study and explanatory variables are often easily available or can be well estimated in measurement error models, see, Gleser $(1992,1993)$ for more details on this aspect. An attempt is made in this paper to express the efficiency properties of all the estimators under consideration as a function of reliability ratios associated with study and explanatory variables only. This helps in obtaining conditions for the superiority of one estimator over the other in terms of reliability ratios only.

Further, most of the literature associated with measurement error models generally assumes the normal distribution for the measurement errors. In practice, such an assumption may not always hold true. The distribution of measurement errors essentially depends on the nature of experiment. The specification of normality may thus sometimes lead to invalid and erroneous statistical consequences. The effect of departure from normality is another aspect of the study which is attempted in this paper.

The finite sample properties of the proposed estimators under different types of distributions of measurement errors are studied through a Monte-Carlo experiment.

The plan of our presentation is as follows. In Section 2, we describe a linear model with measurement errors and present the estimators of the slope parameter when the error variances are known. Section 3 analyzes the asymptotic properties of the estimators when the underlying error distributions are not necessarily normal. The details of the Monte-Carlo experiment and its outcomes are reported in Section 4. Some concluding remarks are offered in Section 5. Lastly, the Appendix outlines the derivation of the main results. 


\section{Model Specification And the Estimators :}

Consider a linear measurement error model in which the variables are related by the linear relation

$$
Y_{j}=\alpha+\beta X_{j} \quad(j=1,2, \ldots, n)
$$

where $Y_{j}$ and $X_{j}$ denote the true but unobserved values of the variables, $\alpha$ is the unknown intercept term and $\beta$ is the unknown slope parameter.

The observed values $y_{j}$ and $x_{j}$ are expressible as

$$
\begin{aligned}
& y_{j}=Y_{j}+u_{j} \\
& x_{j}=X_{j}+v_{j}
\end{aligned}
$$

where $u_{j}$ and $v_{j}$ denote the associated measurement errors.

We assume that $X_{1}, X_{2}, \ldots, X_{n}$ are independently distributed random variables such that $\operatorname{plim}_{n \rightarrow \infty} \bar{X}=: \mu_{X}$ and $\operatorname{plim}_{n \rightarrow \infty} \frac{1}{n} \sum\left(X_{j}-\bar{X}\right)^{2}=: \sigma_{X}^{2}$ exist and $\sigma_{X}^{2}>0$. The measurement errors $u_{1}, u_{2}, \ldots, u_{n}$ are assumed to be independently and identically distributed with mean 0 , variance $\sigma_{u}^{2}$ third moment $\gamma_{1 u} \sigma_{u}^{3}$ and fourth moment $\left(\gamma_{2 u}+3\right) \sigma_{u}^{4}$. The quantities $\gamma_{1}$. and $\gamma_{2}$. represent the measures of skewness and kurtosis of the respective distributions denoted in subscripts. Similarly, the errors $v_{1}, v_{2}, \ldots, v_{n}$ are assumed to be independently and identically distributed with mean 0 , variance $\sigma_{v}^{2}$ third moment $\gamma_{1 v} \sigma_{v}^{3}$ and fourth moment $\left(\gamma_{2 v}+3\right) \sigma_{v}^{4}$. Further, the random variables $\left(X_{j}, u_{j}, v_{j}\right)$ are assumed to be jointly independent.

It may be noted that this model comprises the so-called ultrastructural model, see Dolby (1976), which in turn contains the structural and the functional model as special cases. In the structural model, the $X_{j}$ 's are i.i.d. random variables with mean $\mu_{X}$ and variance $\sigma_{X}^{2}$. In the functional model, the $X_{j}$ are unknown constants with the property that $\lim _{n \rightarrow \infty} \bar{X}=: \mu_{X}$ and $\frac{1}{n} \sum\left(X_{j}-\bar{X}\right)^{2}=: \sigma_{X}^{2}$ exist.

Consistent estimation of the parameters $\alpha$ and $\beta$ in the relationship (2.1) with the help of given data $\left(x_{j}, y_{j}\right), j=1, \ldots, n$, is possible only when some additional 
information is available.

This additional information, let us suppose, specifies the error variances $\sigma_{u}^{2}$ and $\sigma_{v}^{2}$. We can then estimate the slope parameter $\beta$ consistently by the method of moments. This provides the following estimators of $\beta$.

$$
\begin{aligned}
b_{d} & =\frac{s_{x y}}{s_{x x}-\sigma_{v}^{2}} ; s_{x x}>\sigma_{v}^{2} \\
b_{i} & =\frac{s_{y y}-\sigma_{u}^{2}}{s_{x y}} ; s_{y y}>\sigma_{u}^{2}
\end{aligned}
$$

where

$$
\begin{gathered}
s_{x x}=\frac{1}{n} \sum\left(x_{j}-\bar{x}\right)^{2} \quad, \quad \bar{x}=\frac{1}{n} \sum x_{j} ; \\
s_{y y}=\frac{1}{n} \sum\left(y_{j}-\bar{y}\right)^{2} \quad, \quad \bar{y}=\frac{1}{n} \sum y_{j} ; \\
s_{x y}=\frac{1}{n} \sum\left(x_{j}-\bar{x}\right)\left(y_{j}-\bar{y}\right) .
\end{gathered}
$$

When $s_{x x}<\sigma_{v}^{2}$ in $b_{d}$ or $s_{y y}<\sigma_{u}^{2}$ in $b_{i}$, then one possible solution is to disregard the whole sample because the measurement errors are too high to spoil the whole data set. Another alternative is to use the small sample modifications in the estimators as suggested in Fuller (1987). Further discussion on this issue is out of the purview of this paper.

The estimator $b_{d}$ can be regarded as the direct OLS estimator of the slope parameter in the regression of $y_{j}$ on $x_{j}^{*}$ instead of $x_{j}$; see, e.g., Srivastava and Shalabh (1997a) and Shalabh (1998), where

$$
x_{j}^{*}=\bar{x}+\left(1-\frac{\sigma_{v}^{2}}{s_{x x}}\right)\left(x_{j}-\bar{x}\right) .
$$

Similarly, if we write

$$
y_{j}^{*}=\bar{y}+\left(1-\frac{\sigma_{u}^{2}}{s_{y y}}\right)\left(y_{j}-\bar{y}\right)
$$

the inverse estimator $b_{i}$ essentially arises from the regression of $x_{j}$ on $y_{j}^{*}$ instead of $y_{j}$. 
It may be observed that the estimators $b_{d}$ and $b_{i}$ utilize the knowledge of only one error variance at a time. An estimator using the knowledge of both the error variances is given by

$$
b_{p}=t_{p}+\left(t_{p}^{2}+\frac{\sigma_{u}^{2}}{\sigma_{v}^{2}}\right)^{\frac{1}{2}} ; t_{p}=\frac{1}{2 s_{x y}}\left(s_{y y}-\frac{\sigma_{u}^{2}}{\sigma_{v}^{2}} s_{x x}\right) ; s_{x y} \neq 0
$$

which is obtained by minimizing the sum of squares of the perpendicular distance from the data points to the line in a scatter diagram, (i.e., orthogonal regression) after the data $x_{j}$ and $y_{j}$ have been transformed to $x_{j} / \sigma_{v}$ and $y_{j} / \sigma_{u}$, respectively.

In the technique of "reduced major axis", the slope parameter $\beta$ is estimated by the geometric mean of the estimators arising from direct and inverse regression estimators as

$$
b_{g}=\operatorname{sign}\left(s_{x y}\right)\left|b_{d} b_{i}\right|^{\frac{1}{2}},
$$

where $\operatorname{sign}\left(s_{x y}\right)$ is the sign of $s_{x y}$ which can be either positive or negative.

Similarly, we may estimate $\beta$ by

$$
b_{m}=\frac{1}{2}\left(b_{d}+b_{i}\right),
$$

which is the arithmetic mean of estimators $b_{d}$ and $b_{i}$.

Another interesting estimator of $\beta$ is

$$
b_{b}=t_{b}+\left(t_{b}^{2}+1\right)^{\frac{1}{2}} ; t_{b}=\frac{b_{d} b_{i}-1}{b_{d}+b_{i}},
$$

which is the slope of the line that bisects the angle between the two regression lines specified by $b_{d}$ and $b_{i}$.

It may be observed that all six estimators of $\beta$ can be seen to have arisen from the method of moments.

\section{Asymptotic Properties:}

The asymptotic variances of the estimator $b_{d}, b_{i}$ and $b_{p}$ under an ultrastructural model and when errors are not necessarily normally distributed have been studied 
by Shalabh, Gleser and Rosen (2004), see also, Srivastava and Shalabh (1997b), Schneeweiss (1976) and Fuller (1987). For the sake of convenience to the reader, we restate these results. In addition, we give an expression for the asymptotic covariance of $b_{d}$ and $b_{i}$, which will be used in the derivation of the asymptotic variances of $b_{g}, b_{m}$ and $b_{b}$.

Proposition 1: The estimators $b_{d}$ and $b_{i}$ are asymptotically jointly normally distributed as

$$
\sqrt{n}\left(\begin{array}{c}
b_{d}-\beta \\
b_{i}-\beta
\end{array}\right) \rightarrow N\left(0, \Sigma_{b}\right) \quad \text { where } \quad \Sigma_{b}=\left(\begin{array}{cc}
\sigma_{d d} & \sigma_{d i} \\
\sigma_{d i} & \sigma_{i i}
\end{array}\right)
$$

with

$$
\begin{aligned}
\sigma_{d d} & =\beta^{2}\left(\frac{1-\lambda_{x}}{\lambda_{x}^{2}}\right)\left[\lambda_{x}+q+\left(1-\lambda_{x}\right)\left(2+\gamma_{2 v}\right)\right] \\
\sigma_{i i} & =\beta^{2}\left(\frac{1-\lambda_{x}}{\lambda_{x}^{2}}\right)\left[\lambda_{x}+q+q^{2}\left(1-\lambda_{x}\right)\left(2+\gamma_{2 u}\right)\right] \\
\sigma_{d i} & =\beta^{2}\left(\frac{1-\lambda_{x}}{\lambda_{x}^{2}}\right)\left[\lambda_{x}+q\left(2 \lambda_{x}-1\right)\right]
\end{aligned}
$$

where

$$
\begin{aligned}
\lambda_{x} & =\frac{\sigma_{X}^{2}}{\sigma_{x}^{2}}=\frac{\sigma_{X}^{2}}{\sigma_{X}^{2}+\sigma_{v}^{2}} \\
\lambda_{y} & =\frac{\beta^{2} \sigma_{X}^{2}}{\beta^{2} \sigma_{X}^{2}+\sigma_{u}^{2}} \\
q & =\frac{\lambda_{x}\left(1-\lambda_{y}\right)}{\lambda_{y}\left(1-\lambda_{x}\right)}
\end{aligned}
$$

The proof of this Proposition is stated in the Appendix.

Notice that $\lambda_{x}$ and $\lambda_{y}$ are the reliability ratios of the explanatory and study variables in the model. Obviously, $0<\lambda_{x} \leq 1,0 \leq \lambda_{y} \leq 1$, and $q \geq 0$.

Proposition 2: The estimators $b_{p}$ is asymptotically normally distributed as

$$
\sqrt{n}\left(b_{p}-\beta\right) \rightarrow N\left(0, \sigma_{p p}\right)
$$


with asymptotic variance

$$
\sigma_{p p}=\beta^{2}\left(\frac{1-\lambda_{x}}{\lambda_{x}^{2}}\right)\left[\lambda_{x}+q+\frac{q^{2}\left(1-\lambda_{x}\right)}{(q+1)^{2}}\left(\gamma_{2 u}+\gamma_{2 v}\right)\right] .
$$

The proof of the Proposition 2 follows from Shalabh et al. (2004).

Proposition 3: Let $\hat{\beta}_{1}$ and $\hat{\beta}_{2}$ be two consistent, asymptotically jointly normal estimators of $\beta$. Any estimator $\hat{\beta}$ of $\beta$ which is a differentiable and symmetric function $g\left(\hat{\beta}_{1}, \hat{\beta}_{2}\right)$ of $\hat{\beta}_{1}$ and $\hat{\beta}_{2}$ such that $\beta=g(\beta, \beta)$ is consistent and asymptotically normally distributed with an asymptotic variance given by

$$
\sigma_{\hat{\beta}}^{2}=\frac{1}{4}\left(\sigma_{11}+2 \sigma_{12}+\sigma_{22}\right)
$$

where $\Sigma=\left(\sigma_{i j}\right), \quad i, j=1,2$, is the asymptotic covariance matrix of $\left(\hat{\beta}_{1}, \hat{\beta}_{2}\right)$.

The proof of this Proposition is stated in the Appendix.

Thus, using Propositions 1 and 3 , it is seen that the estimators $b_{g}, b_{m}$, and $b_{b}$ are all consistent and asymptotically normal with the same asymptotic variance given by

$$
\beta^{2} \frac{\left(1-\lambda_{x}\right) \delta}{4 \lambda_{x}^{2}}
$$

where

$$
\delta=2\left[q^{2}\left(1-\lambda_{x}\right)+1+\lambda_{x}+2 q \lambda_{x}\right]+\left(1-\lambda_{x}\right)\left(\gamma_{2 v}+q^{2} \gamma_{2 u}\right) .
$$

It is interesting to observe from (3.5) and (3.6) that the skewness of the distributions of measurement errors has no influence on the asymptotic variances of the estimators. It is only the kurtosis that shows its effect. Further, it is seen that the asymptotic variance for each estimator under normality of errors could be quite different when the distributions depart from normality.

It is interesting to note that the estimators $b_{g}, b_{m}$ and $b_{b}$ are equally efficient. Comparing the asymptotic variances, we find that the estimator $b_{p}$ is more efficient than $b_{g}, b_{m}$ or $b_{b}$ if, and only if,

$$
2\left(q^{2}-1\right)^{2} \geq(q-1)\left[(1+3 q) \gamma_{2 v}-q^{2}(q+3) \gamma_{2 u}\right]
$$


Condition (3.7) is clearly satisfied when both measurement errors have mesokurtic (e.g., normal) distributions. The condition also holds true when $q>1$ and $\gamma_{2 u} \geq 0$ and $\gamma_{2 v}<0$.

When either of the measurement errors has a distribution with non-zero coefficient of kurtosis, the inequality (3.7) may not always hold true. So the estimator $b_{p}$ may not necessarily have minimum variance under non-normal distributions of measurement errors.

Next, we compare the asymptotic variances of $b_{g}, b_{m}$ or $b_{b}$ with $b_{d}$ and $b_{i}$. We find that $b_{g}, b_{m}$ or $b_{b}$ are better than $b_{d}$ if, and only if,

$$
3 \gamma_{2 v}-q^{2} \gamma_{2 u}>2(q-3)(q+1)
$$

Condition (3.8) is always satisfied for mesokurtic (e.g., normal) distributions of $u$ and $v$ when $q<3$.

Similarly, $b_{g}, b_{m}$ or $b_{b}$ are better than $b_{i}$ if, and only if,

$$
\gamma_{2 v}-3 q^{2} \gamma_{2 u}<2(3 q-1)(q+1)
$$

Condition (3.9) is always satisfied for mesokurtic (e.g., normal) distributions of $u$ and $v$ when $q>\frac{1}{3}$.

From (3.8) and (3.9), it is clear that the use of two types of additional information to obtain a consistent estimator is not always a good idea, at least asymptotically. Rather, it depends on the values of reliability ratios associated with dependent and independent variables as well as on the degree of peakedness of the distributions of measurement errors to decide which of the information can give better asymptotic results.

\section{Monte Carlo Simulation:}

The asymptotic theory developed in the previous section gives an idea about the behaviour of estimators for large samples only. We conducted a Monte-Carlo sim- 
ulation to study the behaviour of the estimators in finite samples. The following probability distributions of measurement errors are considered to have an idea of the effect of departure from the normal distribution on the efficiency properties of the estimators:

1. normal distribution,

2. $t$-distribution with 6 degrees of freedom,

3. beta distribution $\operatorname{Beta}(4,2)$, and

4. Weibull distribution with shape parameter 1 and scale parameter 2 .

Two data sets of sample sizes $n=40$ (treated as small sample) and $n=400$ (treated as large sample) are considered, for which $\sigma_{X}^{2}=0.08$. The empirical bias (EB) and empirical mean squared error (EMSE) of the estimators $b_{d}, b_{i}, b_{p}, b_{g}, b_{m}$, and $b_{b}$ are computed based on 10000 replications for both the sample sizes and for different combinations of $\lambda_{x}=0.1,0.3,0.5,0.7,0.9$ and $\lambda_{y}=0.1,0.3,0.5,0.7,0.9$ under different distributions of measurement errors. The values of EB and EMSE of these estimators are presented in Tables 1 to 8 and are plotted against $\lambda_{x}$ and $\lambda_{y}$ in 3-dimensional surface plots in Fig. 1 to 8 . It should be noted that the figures employ different scalings on the $Z$-axis. So the behaviour and dependency of EB and EMSE with respect to $\lambda_{x}$ and $\lambda_{y}$ is more clearly visible from the values compiled in the Tables 1 to 8 . On the other hand, the shape of the functions $\operatorname{EB}\left(\lambda_{x}, \lambda_{y}\right)$ and $\operatorname{EMSE}\left(\lambda_{x}, \lambda_{y}\right)$ comes out more clearly in the figures.

The following notations are used in figures 1 to $8: \lambda_{x}=\mathrm{lx}, \lambda_{y}=\mathrm{ly}, b_{d}=\mathrm{bd}$, $b_{i}=\mathrm{bi}, b_{p}=\mathrm{bp}, b_{g}=\mathrm{bg}, b_{m}=\mathrm{bm}$ and $b_{b}=\mathrm{bb}$.

Now we analyze the behaviour of EB and EMSE of different estimators through Fig. 1 to 8 under a given distribution of measurement errors. We are mainly concerned in the pattern of the surfaces rather than the magnitude of values.

First we compare the properties of the various surface plots of EB in Fig. 1 to 4 under small and large samples. Generally speaking, the small sample plots 
$(n=40)$ show much more variability than the large sample plots $(n=400)$. This is particularly true for small $\lambda_{x}$ and $\lambda_{y}$. This variability reflects the extremely high variances of the estimates for small $\lambda_{x}$ and $\lambda_{y}$ (see below). Nevertheless, the bias is statistically significant in most cases.

In the case of normal measurement errors (Fig. 1), the surface plots of EB of $b_{d}$ and $b_{i}$ are similar under small and large samples, whereas the surface plots of $b_{p}$, $b_{g}, b_{m}$, and $b_{b}$ differ between small and large samples. The The EB of $b_{g}$ changes sign when going from small to large samples and when $\lambda_{x}$ and $\lambda_{y}$ are small. Under the $t$ distribution of measurement errors, (see Fig. 2), only the EBs of $b_{g}$ and $b_{b}$ are similar in their behaviour under small and large samples. The sign of EB of $b_{d}$ is partly positive and partly negative in small samples depending on the values of $\lambda_{x}$ and $\lambda_{y}$, whereas it is always negative in large samples. Under the beta distribution of measurement errors, (see Fig.3), only $b_{d}$ has similar surface plots in small and large samples, while the rest shows differences. In small samples, $b_{i}$ and $b_{g}$ are negatively biased either for very low or very high values of $\lambda_{x}$ and $\lambda_{y}$, whereas they are always positively biased in large samples. The EB of $b_{d}$ and $b_{g}$ have similar surface plots in small and large samples under Weibull distributed measurement errors (see Fig. 4). Only $b_{d}$ is negatively biased, whereas the sign of the bias of the other estimators depends on the values of $\lambda_{x}$ and $\lambda_{y}$.

Next, we analyze the dependence of the EMSE of the estimators from Fig. 5 to 8 on the sample size. In general, all EMSE values become small when $\lambda_{x}$ and $\lambda_{y}$ are large, whereas for small $\lambda_{x}$ and/or $\lambda_{y}$, the EMSE often becomes extremely large. Under normally distributed measurement errors (see Fig. 5), the surface plots of $b_{i}$ are quite similar for small and large samples, whereas those for the other estimators show marked differences in small and large samples especially if $\lambda_{x}$ and/ or $\lambda_{y}$ are small. Under the $t$ distribution of measurement errors (see, Fig. 6), no similarity can be seen between small and large samples. Only the surface plots of EMSEs of $b_{d}$ and $b_{i}$ under the beta distributed measurement 
errors (see Fig. 7) are similar between small and large samples. More variation in the corresponding EMSEs of all estimators under small and large samples is seen in Weibull distributed measurement errors (see Fig. 8). None of the estimators have similar surface plots in small and large samples.

Now we compare the different surface plots of EB (Fig. 2 to 4) with those of the normal distribution (Fig. 1) thereby studying the effect of deviations from normality on the EB of the various estimators. Comparing the surface plots of EB of different corresponding estimators under normal and $t$-distributed measurement errors (Fig.1 and 2), we find that all are different except $b_{p}$. The difference is more pronounced in the EBs of corresponding estimators in small samples than in large samples. Such a difference may be seen as the contribution of peakedness of the distribution on EB. The surface patterns of all estimators under normal and beta distributions of measurement errors are remarkably similar (Fig. 1 and 3). The reason being that the coefficients of skewness and kurtosis of the distributions under consideration have very small values. The surface plots of each of the corresponding estimators under normal and Weibull distributions of measurement errors (Fig. 1 and 4) are different except for $b_{p}$ in large samples. This clearly indicates that the departure from normality do affect the EB of these estimators.

Now we compare the surface plots of the empirical mean squared error (EMSE) of different estimators under different distributions of measurement errors (Fig. 6 to 8) to those under normally distributed measurement errors (Fig. 5). Firstly, we compare the surface plots under normal and $t$-distributions of measurement errors (Fig. 5 and 6). We observe that the surface plots of EMSEs of only $b_{i}$ and $b_{p}$ are similar in large samples only and all others are different. This indicates that the kurtosis of the distribution of measurement errors affects the EMSEs of the estimators significantly. Comparison of the surface plots of EMSEs under beta with those under normal distributions of measurement errors (Fig. 8 and 9) reveals that most of the surface plots of estimators are similar because of low 
values of coefficient of skewness and kurtosis of the beta distribution (Fig. 5 and 7). Similarly, comparing the surface plots of EMSEs under Weibull distribution and normal distribution reveals that only $b_{i}, b_{d}$ and $b_{m}$ have similar behaviour with respect to $\lambda_{x}$ and $\lambda_{y}$ in small samples, but the corresponding large sample plots are different. It is worth noting that $b_{p}$ has similar whereas $b_{m}$ has different surface plots in large samples in respective distributions. We note that the asymptotic theory indicates that $b_{g}, b_{m}$ and $b_{b}$ should have similar surface plots. So it is clear that the departure from normality of the distribution of measurement errors play an important role in determining the EMSE of the estimators. However, this is only true for small $\lambda_{x}$ and $\lambda_{y}$.

Now we look at the behaviour of EB and EMSE with respect to $\lambda_{x}$ and $\lambda_{y}$ under different distributions from Tables 1 to 8 . It is observed that the values of EB and EMSE under $n=400$ are closer to the true values of the parameters than under $n=40$ for all distributions, which confirms the consistency of the estimators.

We observe that under the normal distribution of measurement errors (Table 1 and 2) that as either of the values of $\lambda_{x}$ or $\lambda_{y}$ increases, the EB and EMSE of all the estimators decreases. But the magnitude of EB and EMSE of every estimator is different. The $\mathrm{EB}$ of $b_{g}$ has smallest magnitude among other estimators in small samples when $\lambda_{x}$ and $\lambda_{y}$ are low, say, less than 0.3 , otherwise $b_{p}$ has lowest magnitude of EB. An interesting finding is that when $\lambda_{x}$ and $\lambda_{y}$ are very low, the performance of $b_{b}$ is best among other estimators with respect to EMSE. This dominance is stronger in small samples over a wider range of values of $\lambda_{x}$ and $\lambda_{y}$. In fact, both $b_{g}$ and $b_{b}$ have smaller variability than $b_{p}$ when $\lambda_{x}$ and $\lambda_{y}$ are low. The estimator $b_{p}$ outperforms other estimators only when $\lambda_{x}$ and $\lambda_{y}$ are not very low. We know from large sample theory that $b_{g}, b_{b}$ and $b_{m}$ have the same asymptotic variance. We may therefore expect a similar variability for these estimators in large samples. This however holds true only when $\lambda_{x}$ and $\lambda_{y}$ are 
high. In small samples and with lower values of $\lambda_{x}$ and $\lambda_{y}$, this is no more true. The performance of $b_{p}$ is adversely affected when $\lambda_{y}$ is low in comparison to when $\lambda_{x}$ is low. For higher values of $\lambda_{x}$ and $\lambda_{y}$, the performance of all the estimators stabilizes. It can also be seen from Fig. 1 and 6 that for low values of $\lambda_{x}$ and $\lambda_{y}$, say less than 0.5 , the surface plots are changing, whereas when $\lambda_{x}$ and $\lambda_{y}$ are grater than 0.5 , the plots are smooth. The behaviour of different estimators for different values of $\lambda_{x}$ and $\lambda_{y}$, in particular when they are small, varies differently in different distributions.

For the $t$-distribution of measurement errors (Tables 3 and 4 ), $b_{g}$ and $b_{b}$ have smaller magnitude of EB than other estimators in small samples. The estimator $b_{d}$ is always negatively biased, whereas the direction of bias of other estimators depends on the values of $\lambda_{x}$ and $\lambda_{y}$. Comparing the EMSEs, $b_{b}$ dominates all other estimators when $\lambda_{x}$ and $\lambda_{y}$ are low. The dominating range of $\lambda_{x}$ and $\lambda_{y}$ is wider in small samples than in large samples for both the EB and EMSE. The estimator $b_{p}$ is adversely affected by the lower values of $\lambda_{y}$ than $\lambda_{x}$ in small samples. Overall, $b_{b}$ emerges as a good choice when $\lambda_{x}$ is small. When both are high, then $b_{p}$ is a better choice.

Under the beta distribution of measurement errors (Tables 5 and 6), The magnitude of $\mathrm{EB}$ of $b_{g}$ and $b_{b}$ is smaller than of other estimators in small samples but in large samples, $b_{p}$ has smaller magnitude of EB. Comparing the EMSEs, we find that $b_{d}$ has very high EMSE when $\lambda_{x} \leq 0.3$, whereas $b_{i}, b_{p}$ and $b_{m}$ have high EMSE for $\lambda_{x} \leq 0.3$ or $\lambda_{y} \leq 0.3$. The estimator $b_{g}$ and $b_{b}$ have relatively much lower EMSE than other estimators in small samples. This remains true in large samples only when $\lambda_{x}$ is very low, say, 0.1. Among all estimators, $b_{b}$ has smalle EMSE for lower values of $\lambda_{x}$ and $\lambda_{y}$. The estimator $b_{p}$ outperforms $b_{g}$ and $b_{b}$ only when $\lambda_{x}$ and $\lambda_{y}$ are high in small samples and that over a wide range of values of $\lambda_{x}$ and $\lambda_{y}$. In large sample, this remains true over a narrower range of values of $\lambda_{x}$ and $\lambda_{y}$. Again, $b_{p}$ is severely affected by the lower values of $\lambda_{y}$ in small sample. 
As $\lambda_{x}$ and $\lambda_{y}$ increase, the performance of all the estimators stabilize. In large samples, this stabilization comes much faster than in small samples.

Now we consider Tables 7 and 8. The values of the magnitude of EBs and EMSEs of all the estimators are higher under Weibull distributed measurement errors than the corresponding values under the other distributions. This effect is essentially due to the presence of extreme values in the sample and the shape of the Weibull distribution considered here, which is a J-shaped curve. In spite of this, $b_{b}$ shows its clear dominance with respect to the magnitude of EB and EMSE over $b_{p}$ and $b_{g}$ in small sample when $\lambda_{x} \leq 0.9$ and $\lambda_{y} \leq 0.3$. In case of large samples, this dominance is still present, but the ranges of $\lambda_{x}$ and $\lambda_{y}$ shrink. Under similar conditions, $b_{g}$ emerges as the second best choice of estimator. The performance of $b_{i}$ and $b_{p}$ is more severely affected by the lower values of $\lambda_{y}$ than by the lower values of $\lambda_{x}$. The difference in the values of magnitude of EBs and EMSEs under small and large samples is higher under Weibull distributed measurement errors than under other distributions considered earlier. It can be noticed here that the performance of $b_{p}$ under this case is worst among other cases considered earlier. Still $b_{b}$ and $b_{g}$ turns out to be more robust than $b_{p}$, and $b_{b}$ emerges as winner.

\section{Conclusions :}

We considered six estimators $b_{d}, b_{i}, b_{p}, b_{g}, b_{m}$, and $b_{b}$ for the slope parameter $\beta$ when the error variances $\sigma_{u}^{2}$ and $\sigma_{v}^{2}$ are known in a linear ultrastructural model. These estimators can be regarded as arising from the method of moments. All these estimators are found to be consistent and asymptotically normally distributed.

When the distributions of errors depart from normality, it is interesting to observe that the asymptotic variances of the estimators are influence by the peakedness, and not the asymmetry, of the error distributions. Further it is seen that 
the superiority of an estimator over another under the popular specification of normality may not necessarily carry over when the distributions depart from normality. For example, the uniform superiority of the estimator $b_{p}$ over $b_{g}, b_{m}$, and $b_{b}$ is perturbed when the distributions are not normal and in particular platykurtic.

A study from a Monte Carlo simulation experiment gives an insight into the finite sample properties of the estimators and the effect of departure from normality on the efficiency properties of the estimators. It is clear from the study that the efficiency properties of the estimators are affected by non-normality of the distribution of measurement errors. In particular, the effect of peakedness is clearly seen, which is more pronounced in small samples than in large samples. For large samples, most of the estimators show a behaviour that corresponds to the asymptotic theory, at least for higher values of reliability ratios. As a rule of thumb, the reliability ratios can be said to be large when $\lambda_{x} \geq 0.5$ and $\lambda_{y} \geq 0.5$. In particular, when $n, \lambda_{x}$, and $\lambda_{y}$ are large, then $b_{p}$ is best. Also, in this case $b_{g}$, $b_{m}$, and $b_{b}$ are almost similar, which is in accordance with the asymptotic theory. For lower values of the reliability ratios, there is no unique dominance of any of the estimators. The uniform superiority of $b_{p}$ is questionable when the values of the reliability ratios are very low under not necessarily normally distributed measurement errors. In most of the cases, $b_{b}$ emerges as a better choice than $b_{p}$ when $\lambda_{x}$ and $\lambda_{y}$ are low (although otherwise $b_{p}$ dominates). If $\lambda_{x}$ and $\lambda_{y}$ are small, very large samples are needed to produce results similar to asymptotic theory; $n=400$ is not large enough. It is revealed from the simulation study that the choice of a good estimator depends on the values of the reliability ratios as well as the distribution of measurement errors under consideration. 


\section{Appendix}

Using the fundamental relations of the model (2.1)-(2.3), we find with some algebra

$$
\sqrt{n}\left(\hat{\beta}_{d}-\beta\right)=\frac{\sqrt{n}}{s_{x}^{2}-\sigma_{v}^{2}} Z_{d}
$$

where

$$
Z_{d}=s_{X u}+s_{u v}-\beta s_{X v}-\beta\left(s_{v}^{2}-\sigma_{v}^{2}\right)
$$

Denoting the centralised variable $X$ by $X^{*}=X-\mu_{X}$, we obtain

$$
\sqrt{n} Z_{d}=\frac{1}{\sqrt{n}} \sum_{j=1}^{n}\left[X_{j}^{*} u_{j}+u_{j} v_{j}-\beta X_{j}^{*} v_{j}-\beta\left(v_{j}^{2}-\sigma_{v}^{2}\right)\right]+o_{p}(1) .
$$

Similarly,

$$
\sqrt{n}\left(\hat{\beta}_{i}-\beta\right)=\frac{\sqrt{n}}{s_{x y}} Z_{i}
$$

with

$$
\sqrt{n} Z_{i}=\frac{1}{\sqrt{n}} \sum_{j=1}^{n}\left[\beta X_{j}^{*} u_{j}-\beta u_{j} v_{j}-\beta^{2} X_{j}^{*} v_{j}+u_{j}^{2}-\sigma_{u}^{2}\right]+o_{p}(1) .
$$

By the central limit theorem,

$$
\sqrt{n}\left(\begin{array}{c}
Z_{d} \\
Z_{i}
\end{array}\right) \rightarrow N\left(0, \Sigma_{Z}\right), \quad \text { where } \Sigma_{Z}=\left(\begin{array}{cc}
\sigma_{Z_{d} Z_{d}} & \sigma_{Z_{d} Z_{i}} \\
\sigma_{Z_{d} Z_{i}} & \sigma_{Z_{i} Z_{i}}
\end{array}\right)
$$

with

$$
\begin{aligned}
\sigma_{Z_{d} Z_{i}} & =\sigma_{X}^{2} \sigma_{u}^{2}+\sigma_{u}^{2} \sigma_{v}^{2}+\beta^{2} \sigma_{X}^{2} \sigma_{v}^{2}+\beta^{2}\left(E\left(v^{4}\right)-\sigma_{v}^{4}\right) \\
\sigma_{Z_{i} Z_{i}} & =\beta^{2} \sigma_{X}^{2} \sigma_{u}^{2}+\beta^{2} \sigma_{u}^{2} \sigma_{v}^{2}+\beta^{4} \sigma_{X}^{2} \sigma_{v}^{2}+E\left(u^{4}\right)-\sigma_{u}^{4} \\
\sigma_{Z_{d} Z_{i}} & =\beta \sigma_{X}^{2} \sigma_{u}^{2}-\beta \sigma_{u}^{2} \sigma_{v}^{2}+\beta^{3} \sigma_{X}^{2} \sigma_{v}^{2} .
\end{aligned}
$$

As $\operatorname{plim}_{n \rightarrow \infty}\left(s_{x}^{2}-\sigma_{v}^{2}\right)=\sigma_{X}^{2}$ and $\operatorname{plim}_{n \rightarrow \infty} s_{x y}=\beta \sigma_{X}^{2}$, we finally obtain

$$
\begin{aligned}
\sigma_{d d} & =\frac{1}{\sigma_{X}^{4}}\left[\sigma_{u}^{2}\left(\sigma_{X}^{2}+\sigma_{v}^{2}\right)+\beta^{2} \sigma_{X}^{2} \sigma_{v}^{2}+\beta^{2}\left(\gamma_{2 v}+2\right) \sigma_{v}^{4}\right] \\
\sigma_{i i} & =\frac{1}{\sigma_{X}^{4}}\left[\sigma_{u}^{2}\left(\sigma_{X}^{2}+\sigma_{v}^{2}\right)+\beta^{2} \sigma_{X}^{2} \sigma_{v}^{2}+\frac{1}{\beta^{2}}\left(\gamma_{2 u}+2\right) \sigma_{u}^{4}\right] \\
\sigma_{d i} & =\frac{1}{\sigma_{X}^{4}}\left[\sigma_{u}^{2}\left(\sigma_{X}^{2}-\sigma_{v}^{2}\right)+\beta^{2} \sigma_{X}^{2} \sigma_{v}^{2}\right]
\end{aligned}
$$


Using the definitions of $\lambda_{x}$ and $q$, one can see the equivalence of these expressions to the corresponding ones in Proposition 1.

\section{Proof of Proposition 3:}

Denote the partial derivatives of $g$ with respect to the first and second argument of $g$ by $g_{1}$ and $g_{2}$, respectively. Then by the symmetry of $g$, the equation $\beta=g(\beta, \beta)$ implies

$$
1=g_{1}(\beta, \beta)+g_{2}(\beta, \beta)=2 g_{1}(\beta, \beta)=2 g_{2}(\beta, \beta),
$$

i.e., $g_{1}(\beta, \beta)=g_{2}(\beta, \beta)=\frac{1}{2}$. We then can evaluate $\sigma_{\hat{\beta}}^{2}$ by the delta-method as

$$
\sigma_{\hat{\beta}}^{2}=\frac{1}{4}(1,1) \Sigma(1,1)^{\prime}
$$

which is the statement of Proposition 3.

\section{Acknowledgement}

The second author acknowledges the support from a SERC project from the Department of Science and Technology, Government of India and SFB 386, University of Munich (Germany).

\section{References}

Aaronsom, M., G. Bothum, J. Moultd, J. Huchra, R.A. Schommer and M.E. Cornell (1986): 'A distance scale from the infrared magnitude /HI velocity-width relation. $\nu$. distance moduli to 10 galaxy clusters, and positive detection of bulk supercluster motion toward the microwave anisotrophy', Astrophysical Journal, 302, pp. 536-563.

Cheng, C.L. and J.W. Van Ness (1999): Statistical Regression with Measurement Errors, Arnold Publishers. 
Dorff, M. and J. Gurland (1961): 'Estimation of parameters of a linear functional relation', Journal of the Royal Statistical Society, Series B, Vol. 23, No. 1, pp. 160-170.

Gleser, L.J. (1992): 'The importance of assessing measurement reliability in multivariate regression', Journal of American Statistical Association, 87, 419, pp. 696-407.

Gleser, L.J. (1993): 'Estimation of slope in linear errors-in-variables regression models when the predictors have known reliability matrix', Statistics and Probability Letters, 17 (2), pp. 113-121.

Fuller, W.A. (1987): Measurement Error Models, John Wiley.

Pierce, M.J. and R.B. Tully (1988): 'Distances to virgo and ursa major clusters and a determination of $H_{0}{ }^{\prime}$, Astrophysical Journal, 330, pp. $579-595$.

Schneeweiss, H. (1976): 'Consistent estimation of a regression with errors in the variables', Metrika, 23, pp. 101-115.

Shalabh (1998): 'Improved estimation in measurement error models through Stein-rule procedure', Journal of Multivariate Analysis, 67, 35-48.

Corrigendum : Journal of Multivariate Analysis, 74, p. 162, (2000).

Shalabh, L.J. Gleser, and O. Rosen (2004): 'On the usefulness of knowledge of error variances in the consistent estimation of an unreplicated ultrastructural model', Journal of Statistical Computational \& Simulation, 74, 6 pp. 391-417.

Sokal, R.R. and F.J. Rohalf (1981): Biometry: The principal and Practice of Statistics in Biological Research, Second Edition, Freeman. 
Srivastava, A.K. and Shalabh (1997 a): 'A new property of Stein procedure in measurement error model', Statistics $\&$ Probability Letters, 32, pp. 231-234.

Srivastava, A.K. and Shalabh (1997b): 'Consistent estimation for the non-normal ultrastructural model', Statistics 85 Probability Letters, 34, pp. 67-73. 


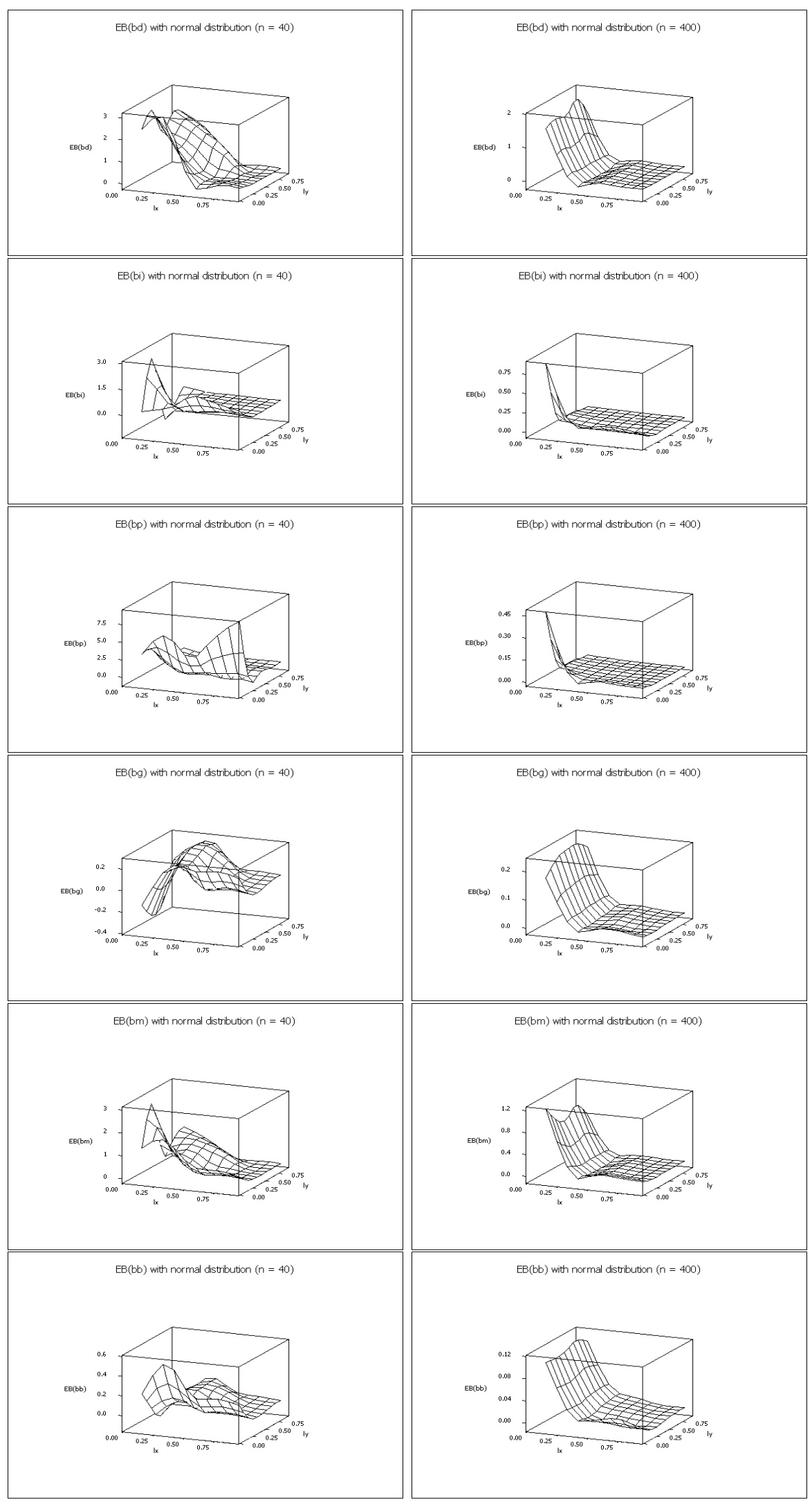

21

Figure 1: Empirical bias of estimators when measurement errors follow normal distribution 


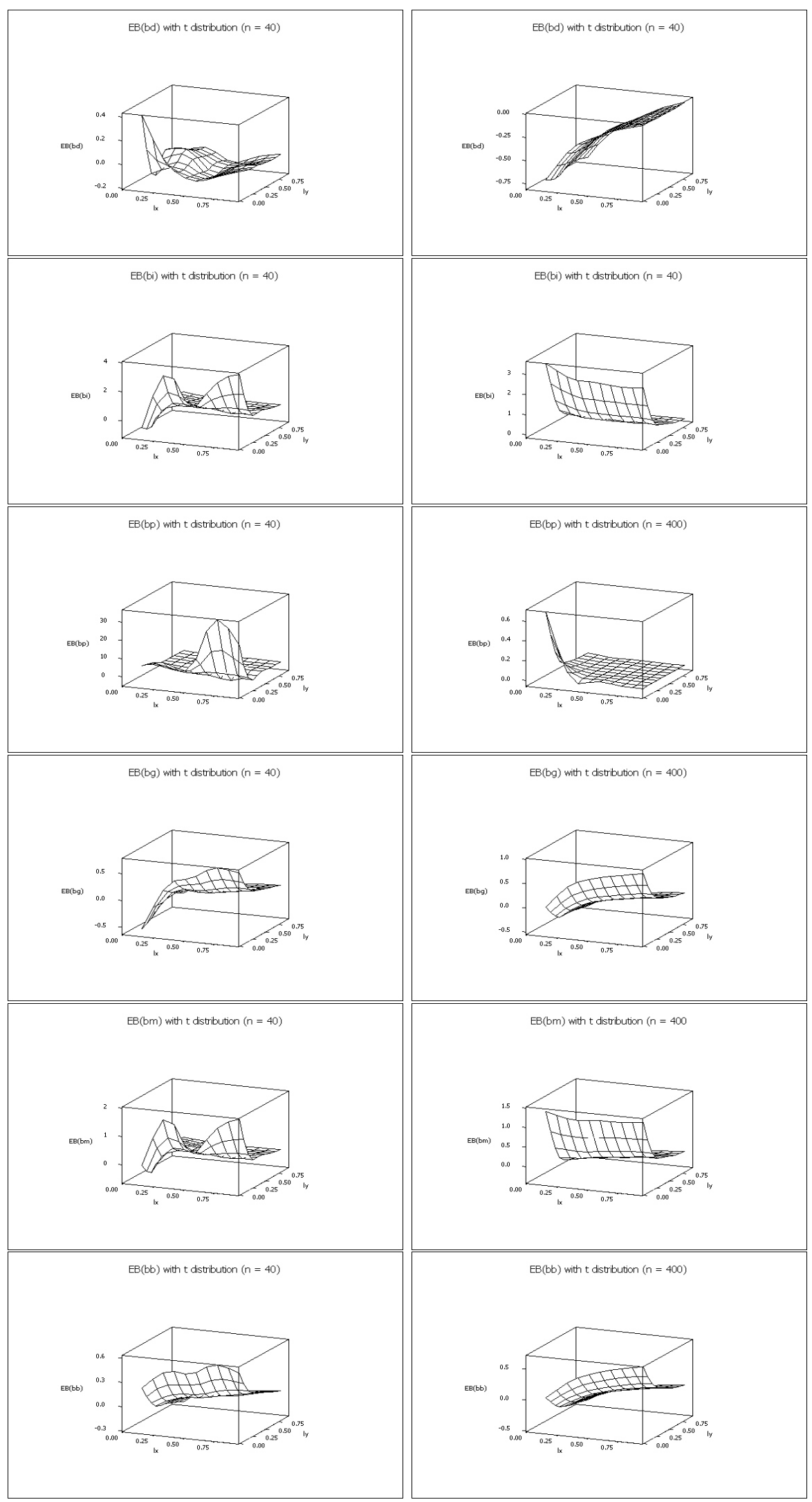

22

Figure 2: Empirical bias of estimators when measurement errors follow $t$ - distribution 


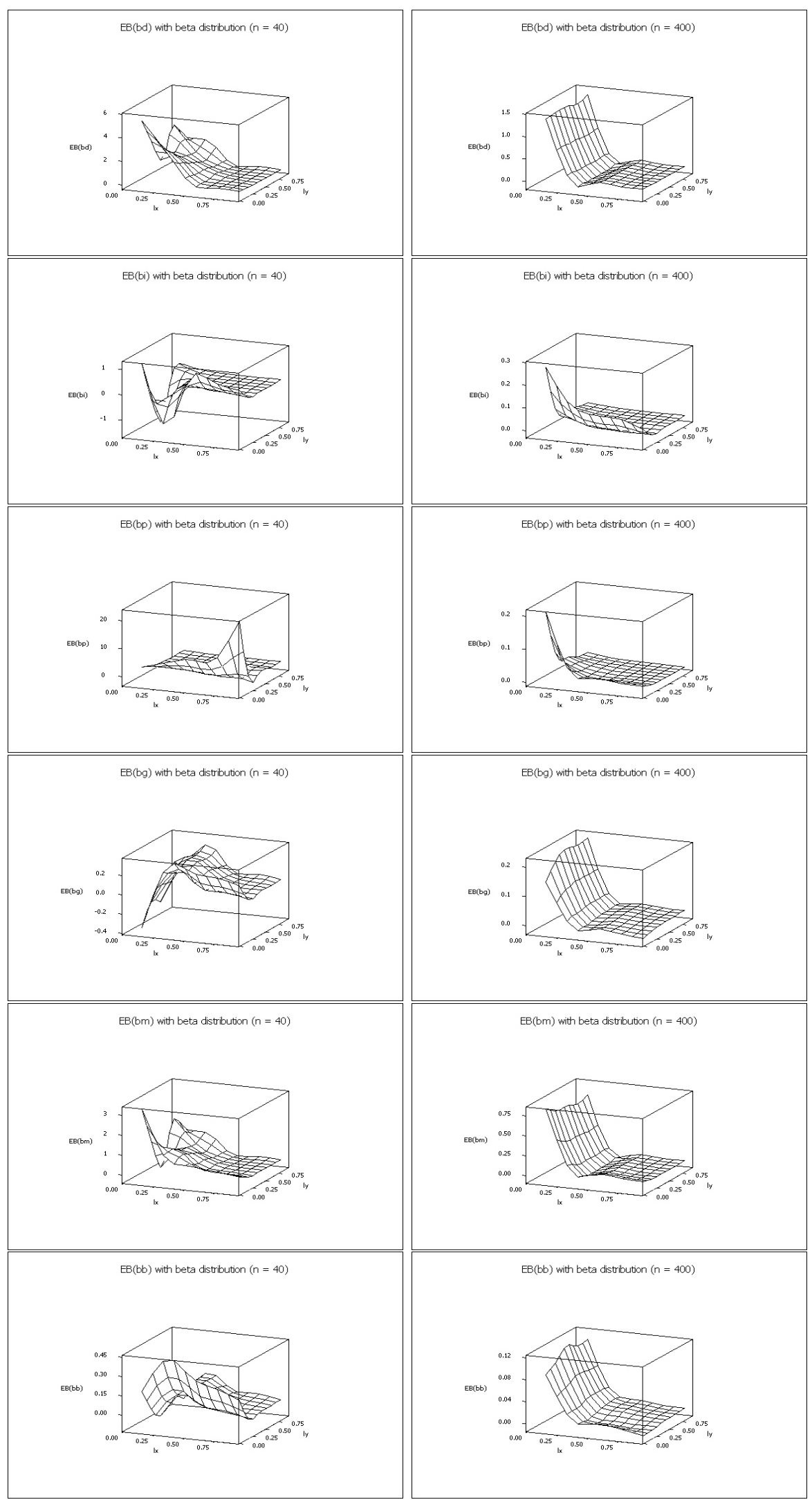

23

Figure 3: Empirical bias of estimators when measurement errors follow beta distribution 


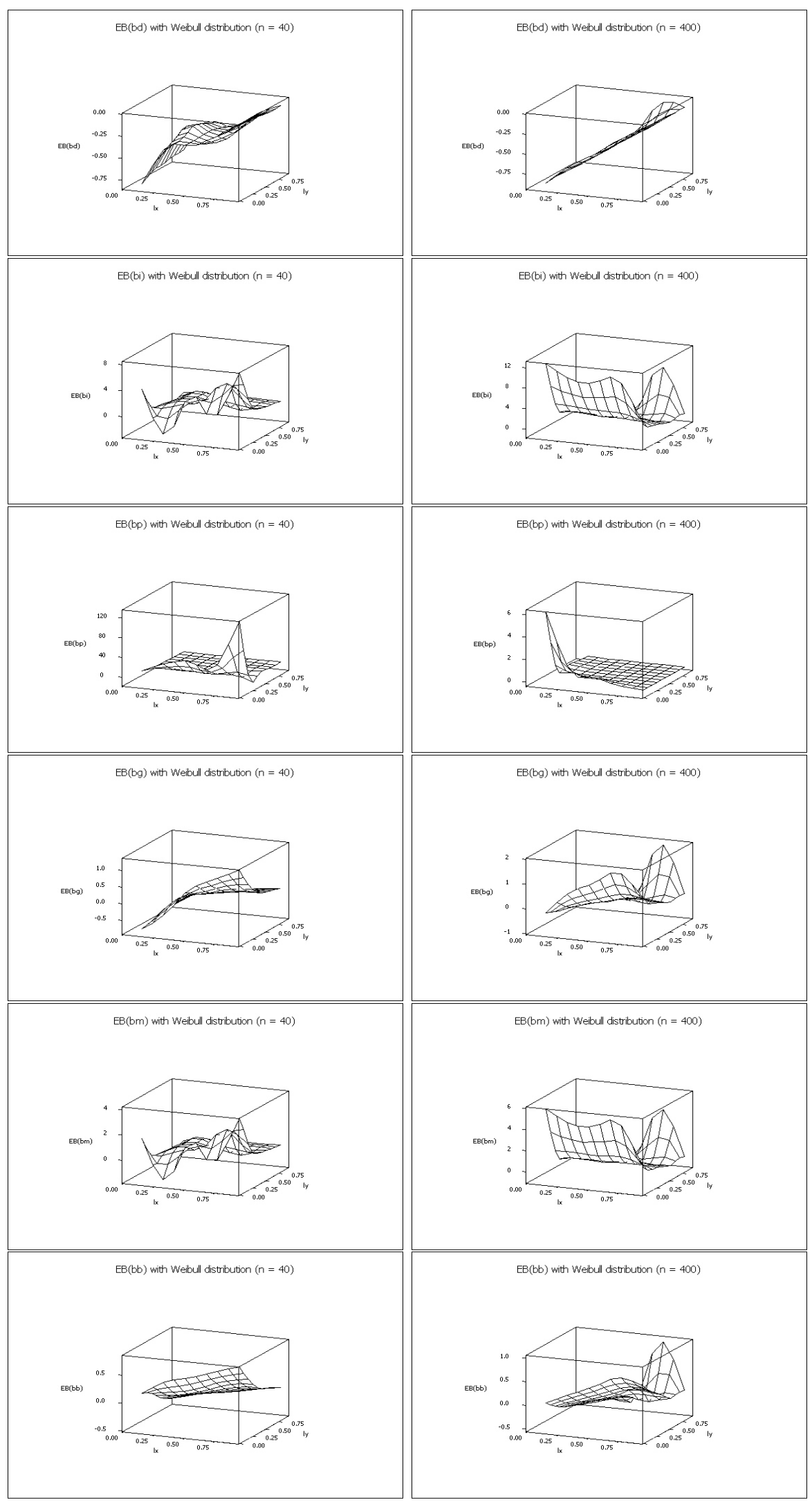

24

Figure 4: Empirical bias of estimators when measurement errors follow Weibull distribution 


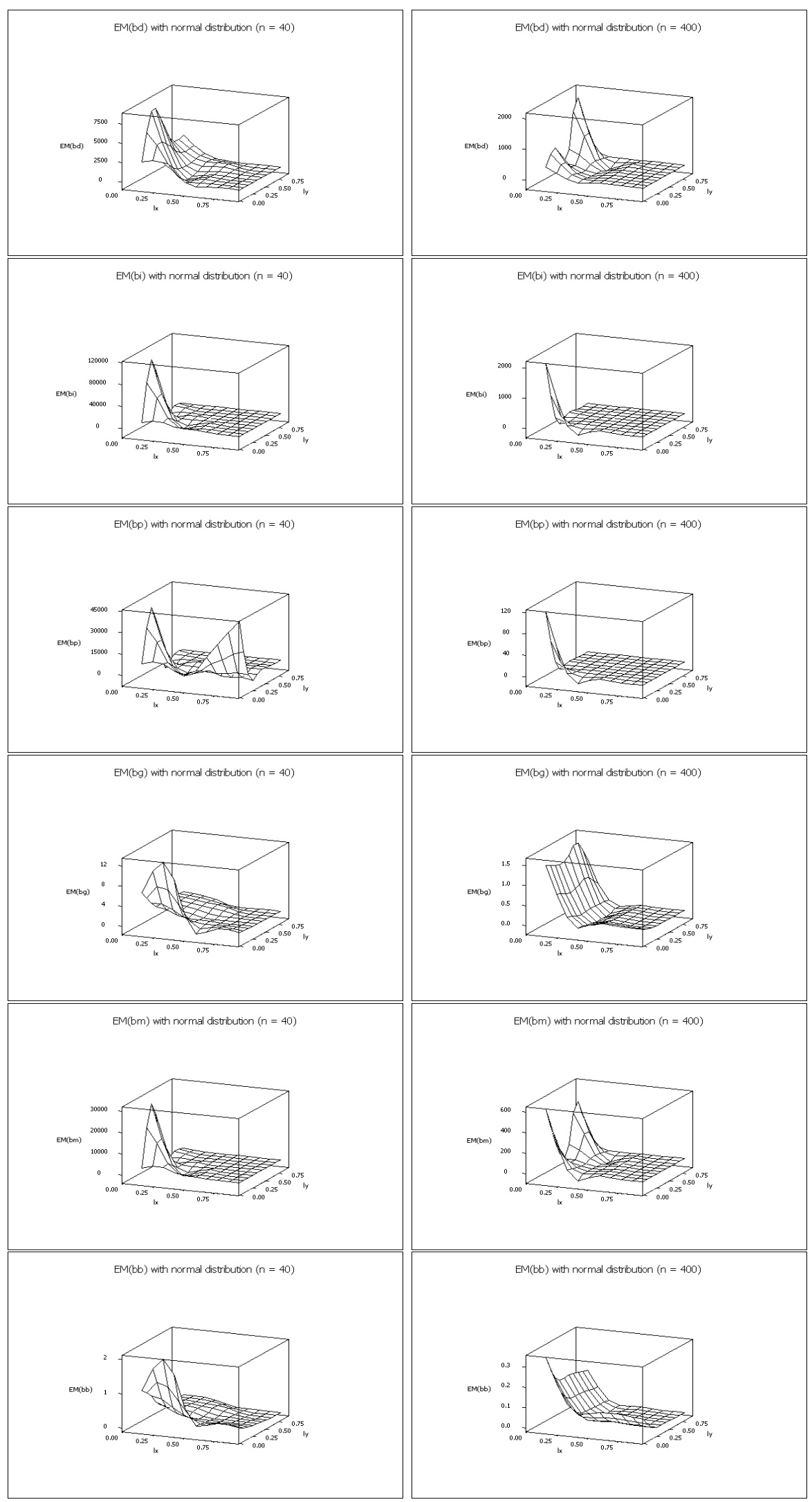

25

Figure 5: Empirical mean squared error of estimators when measurement errors follow normal distribution 


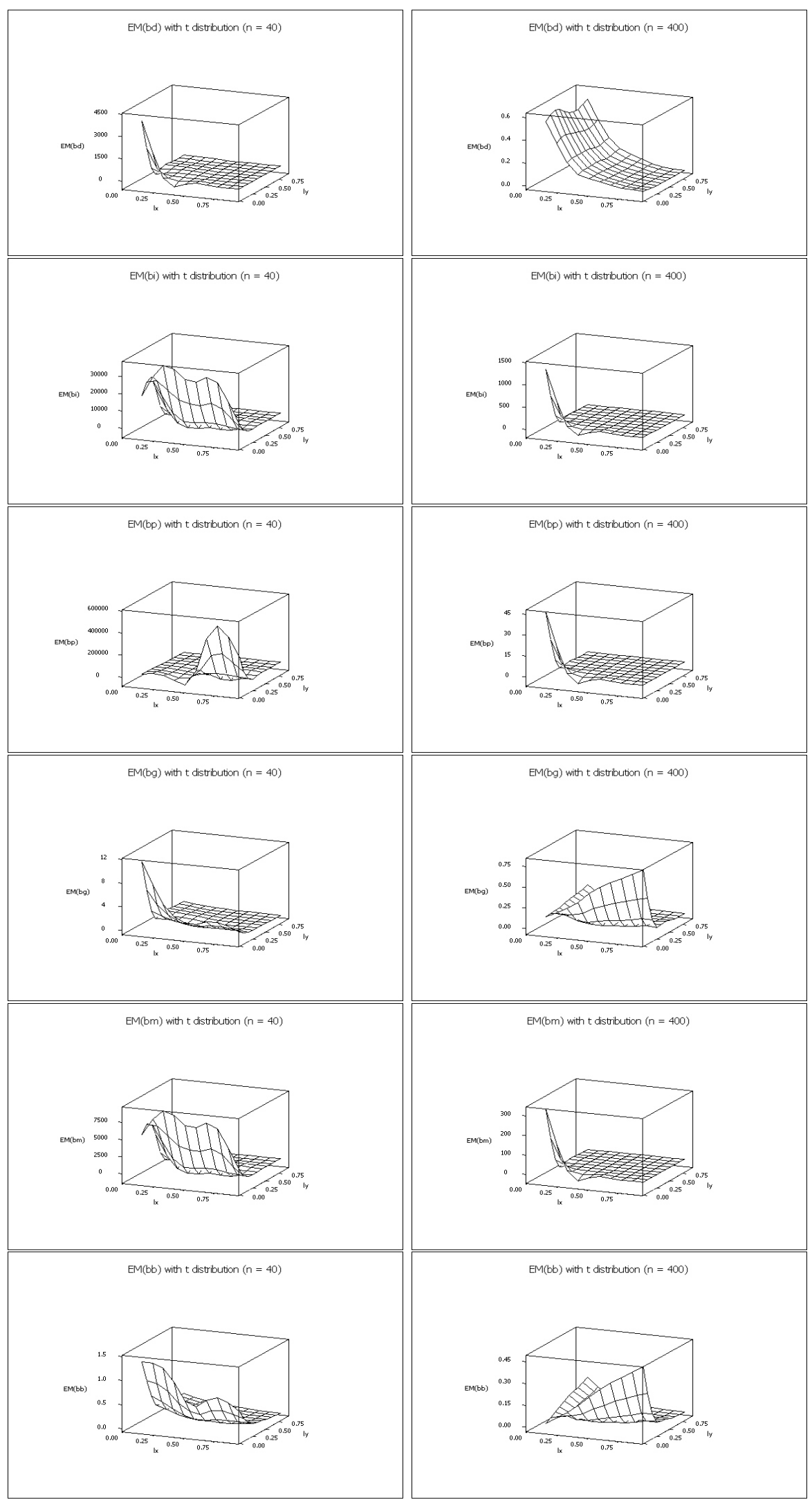

26

Figure 6: Empirical mean squared error of estimators when measurement errors follow $t$ - distribution 


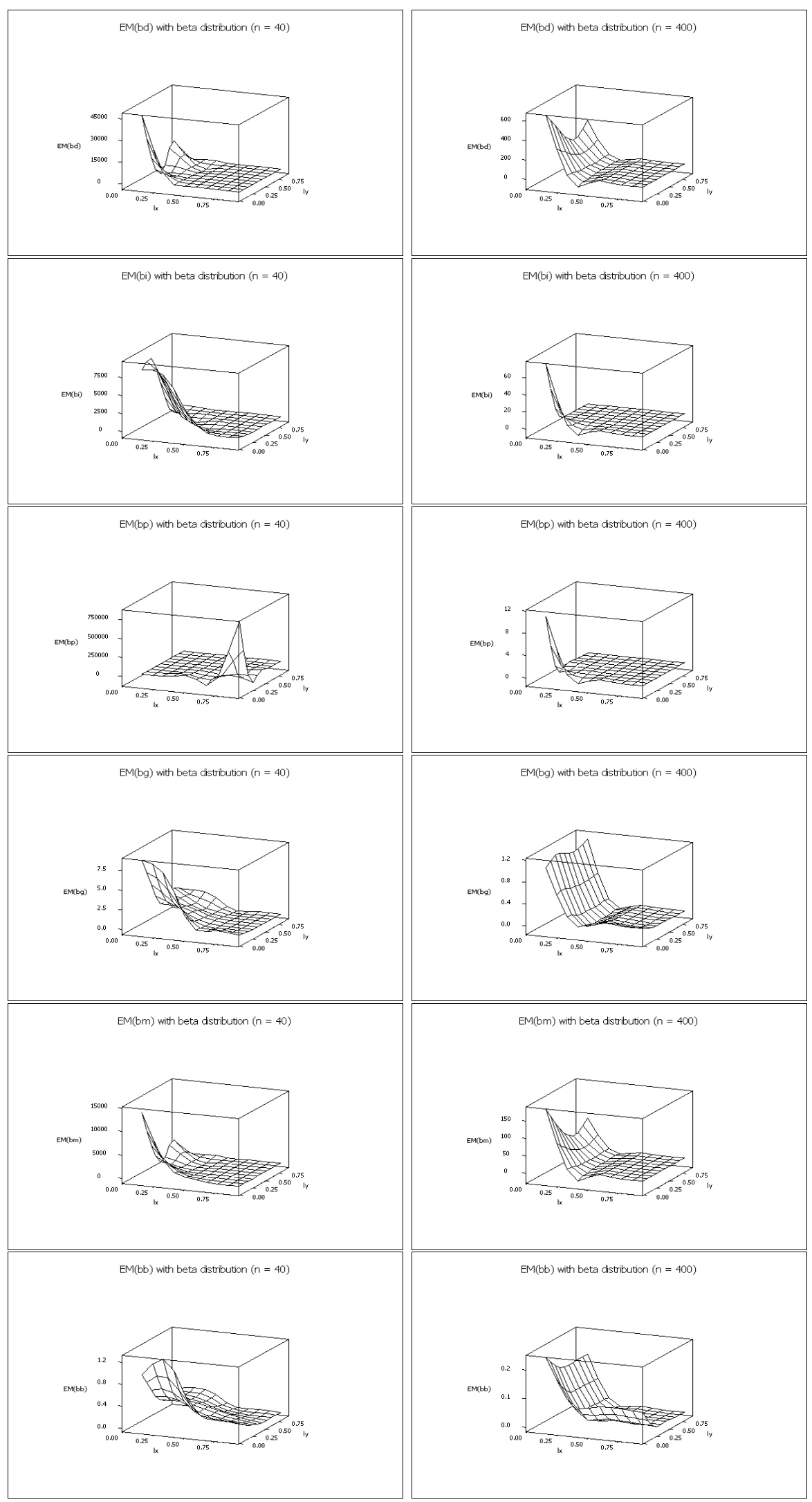

27

Figure 7: Empirical mean squared error of estimators when measurement errors follow beta distribution 


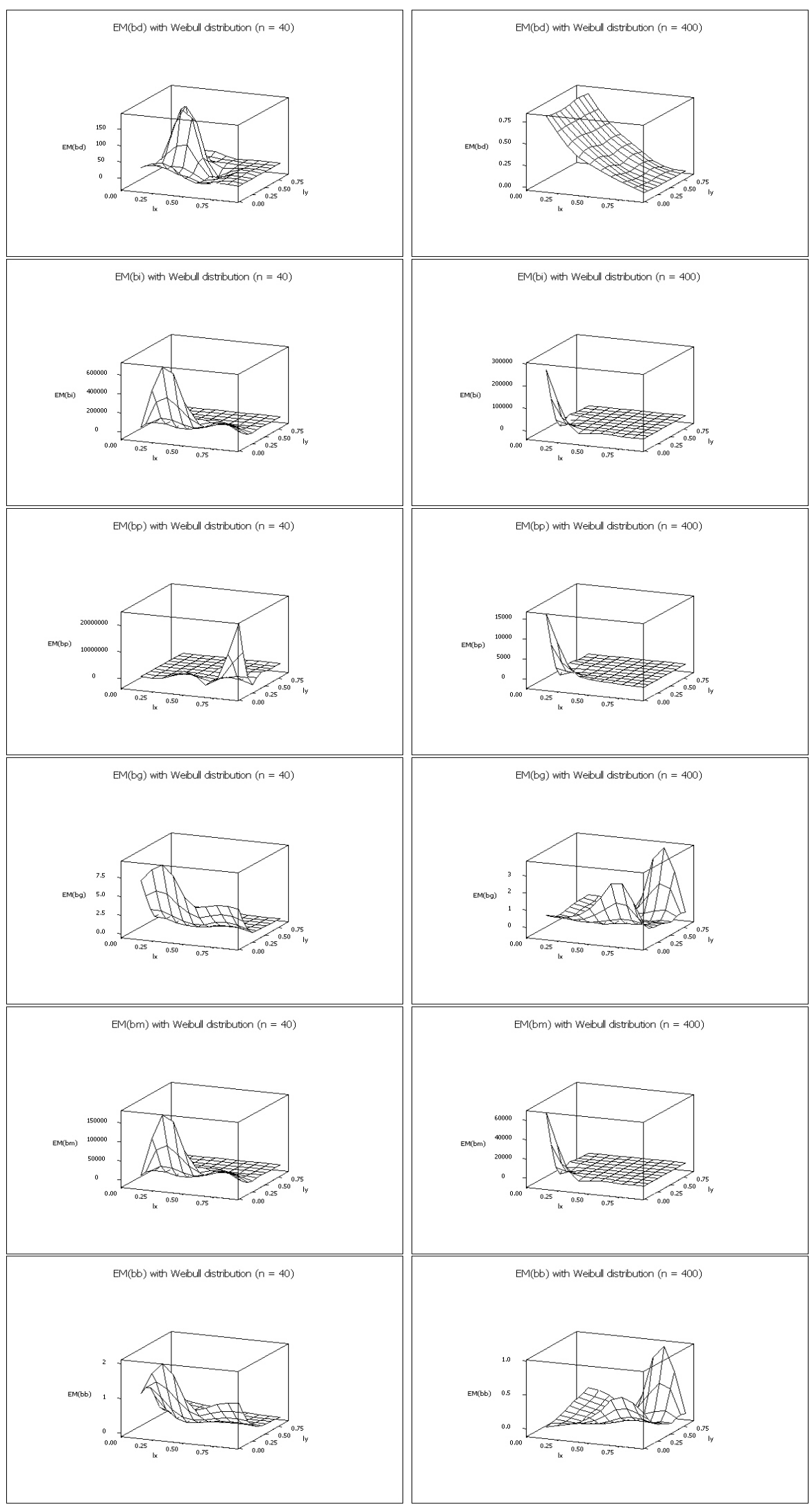

Figure 8: Empirical mean squared error of estimators when measurement errors follow Weibull distribution 


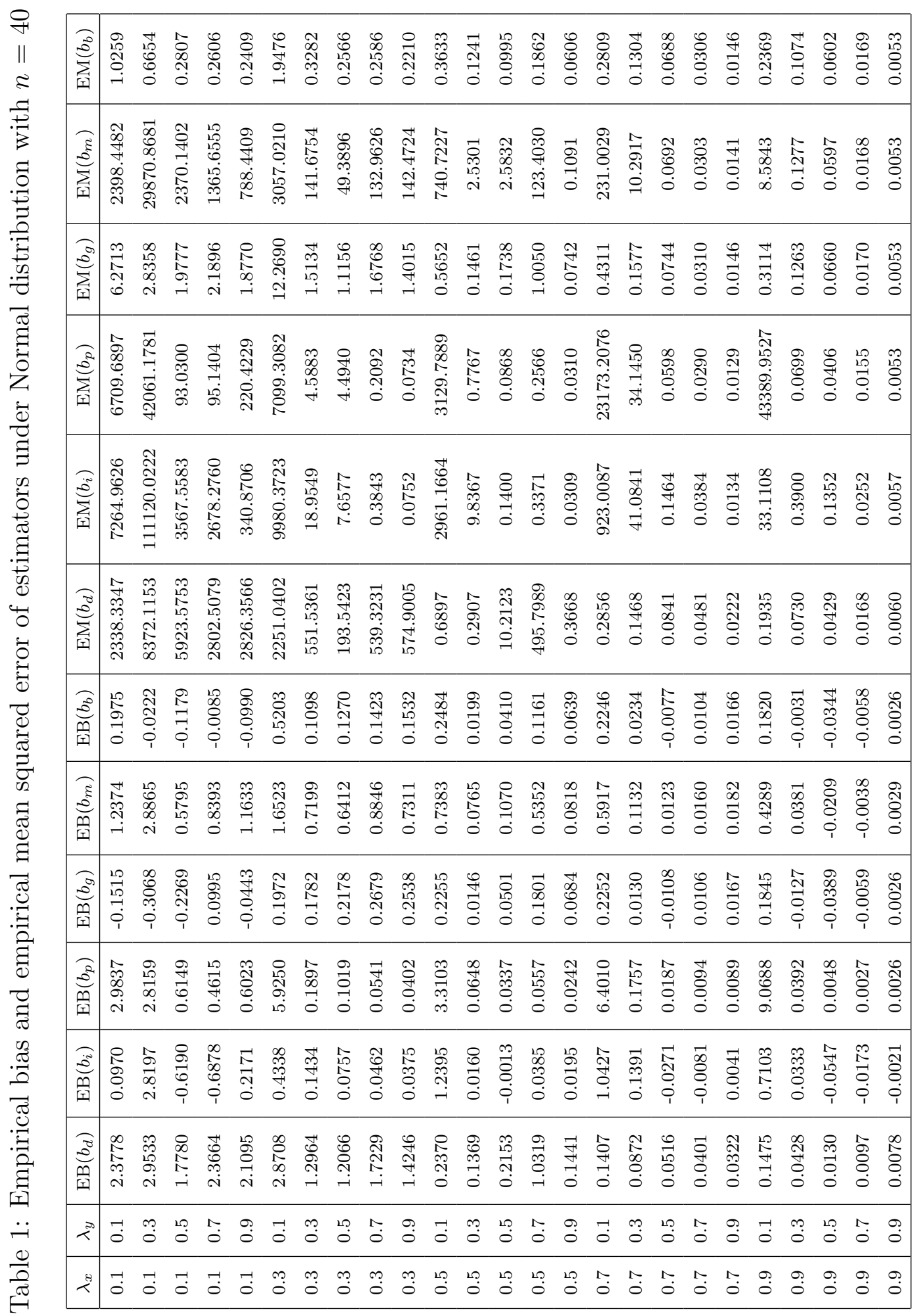




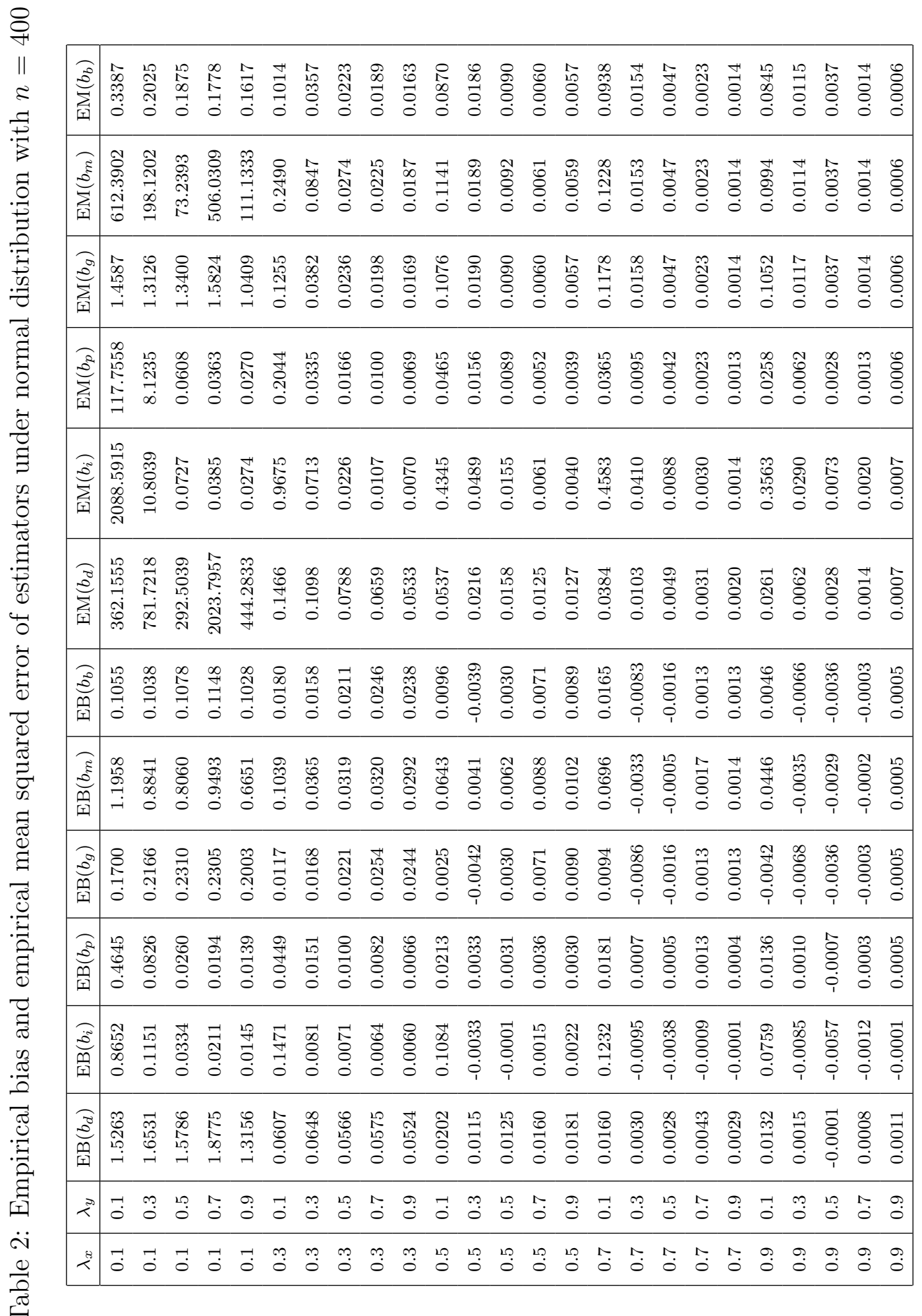




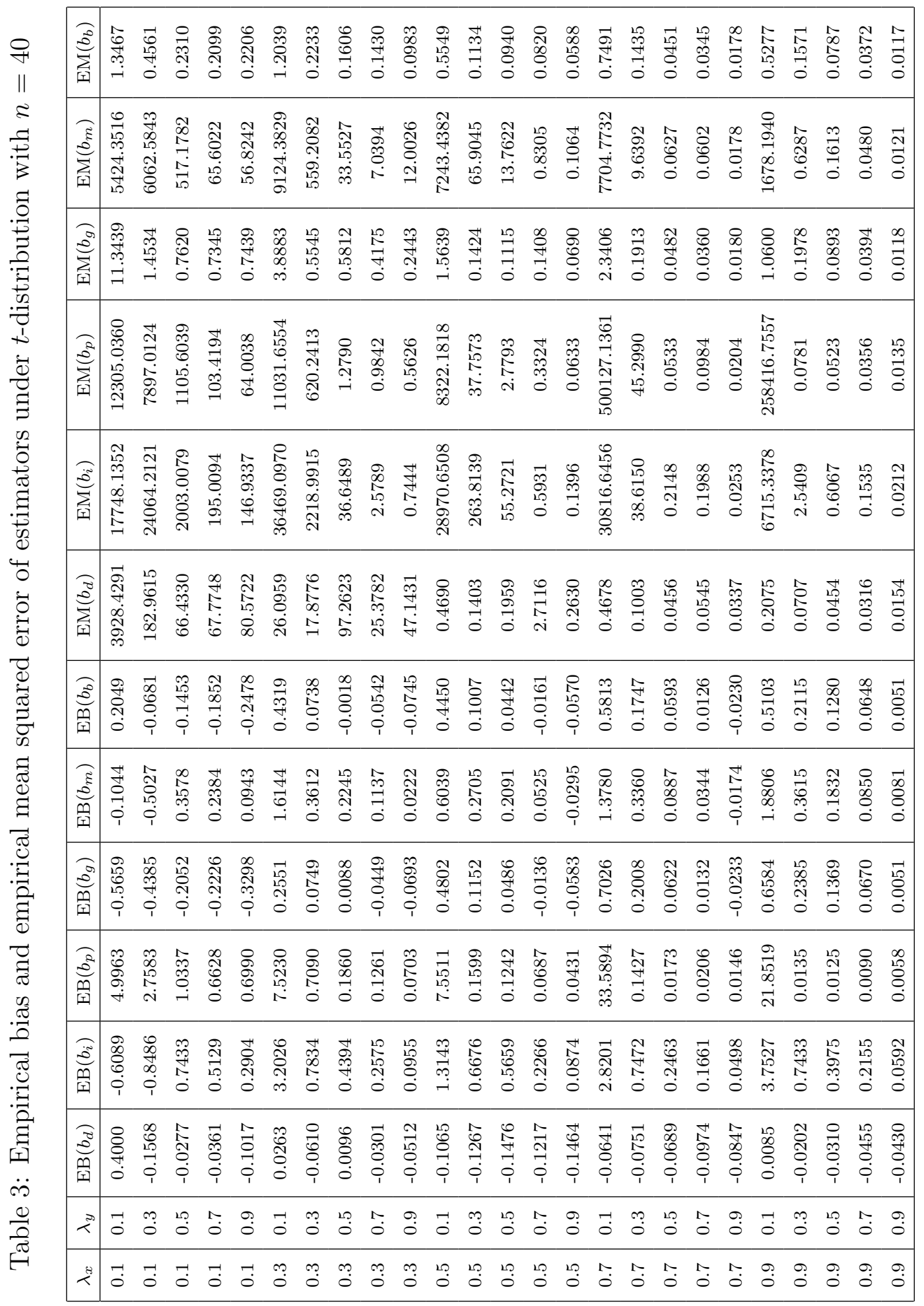




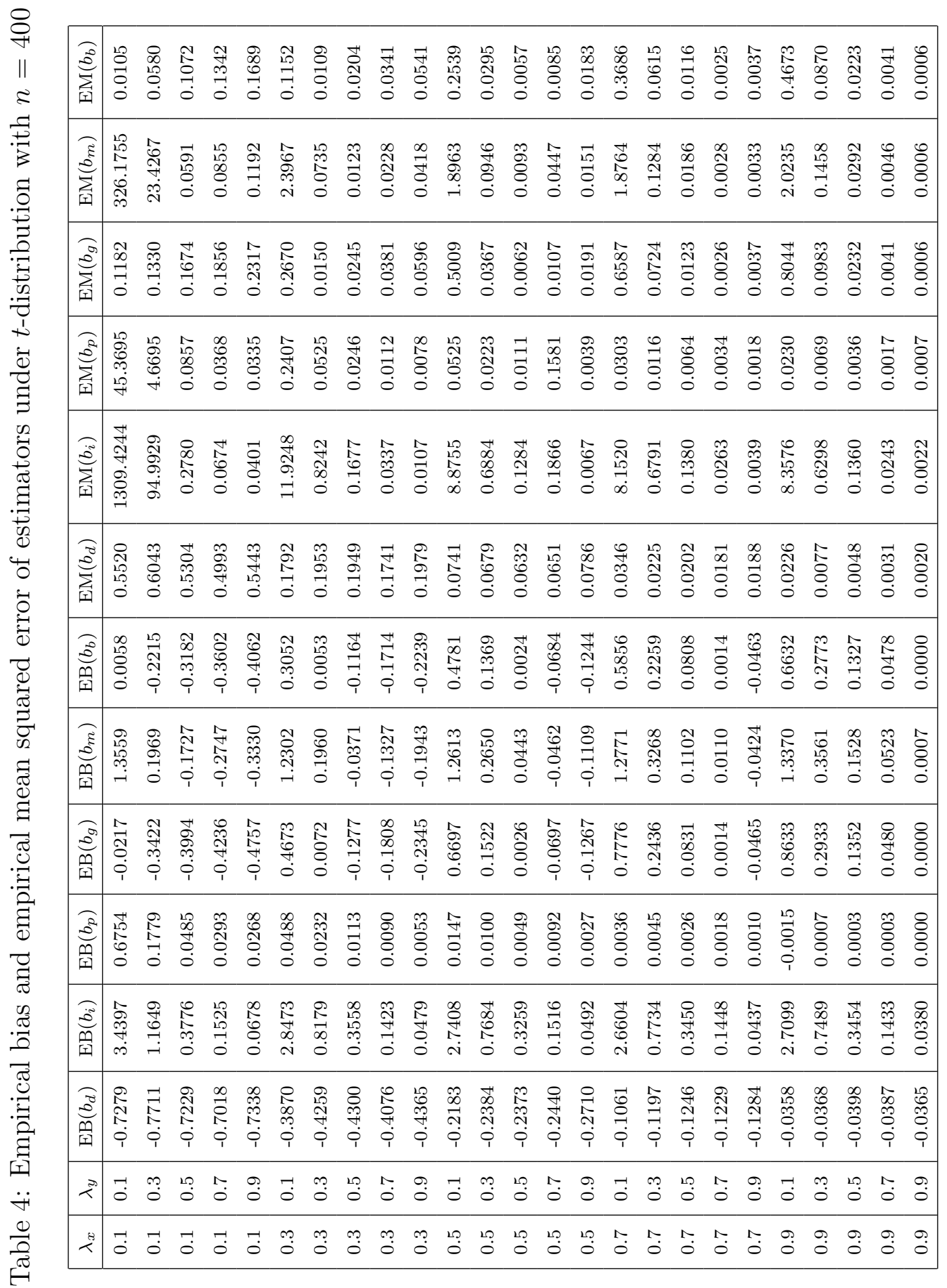




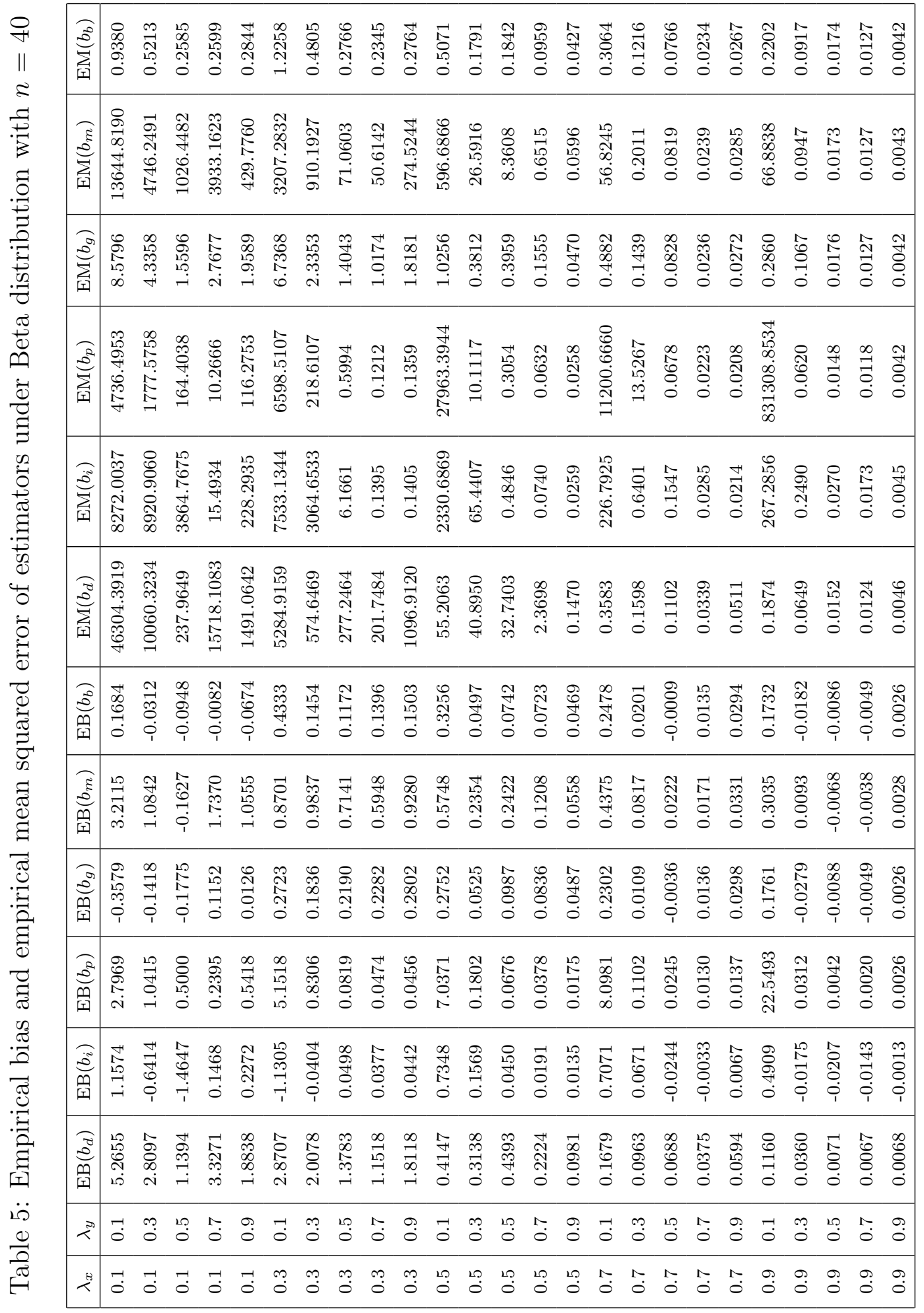




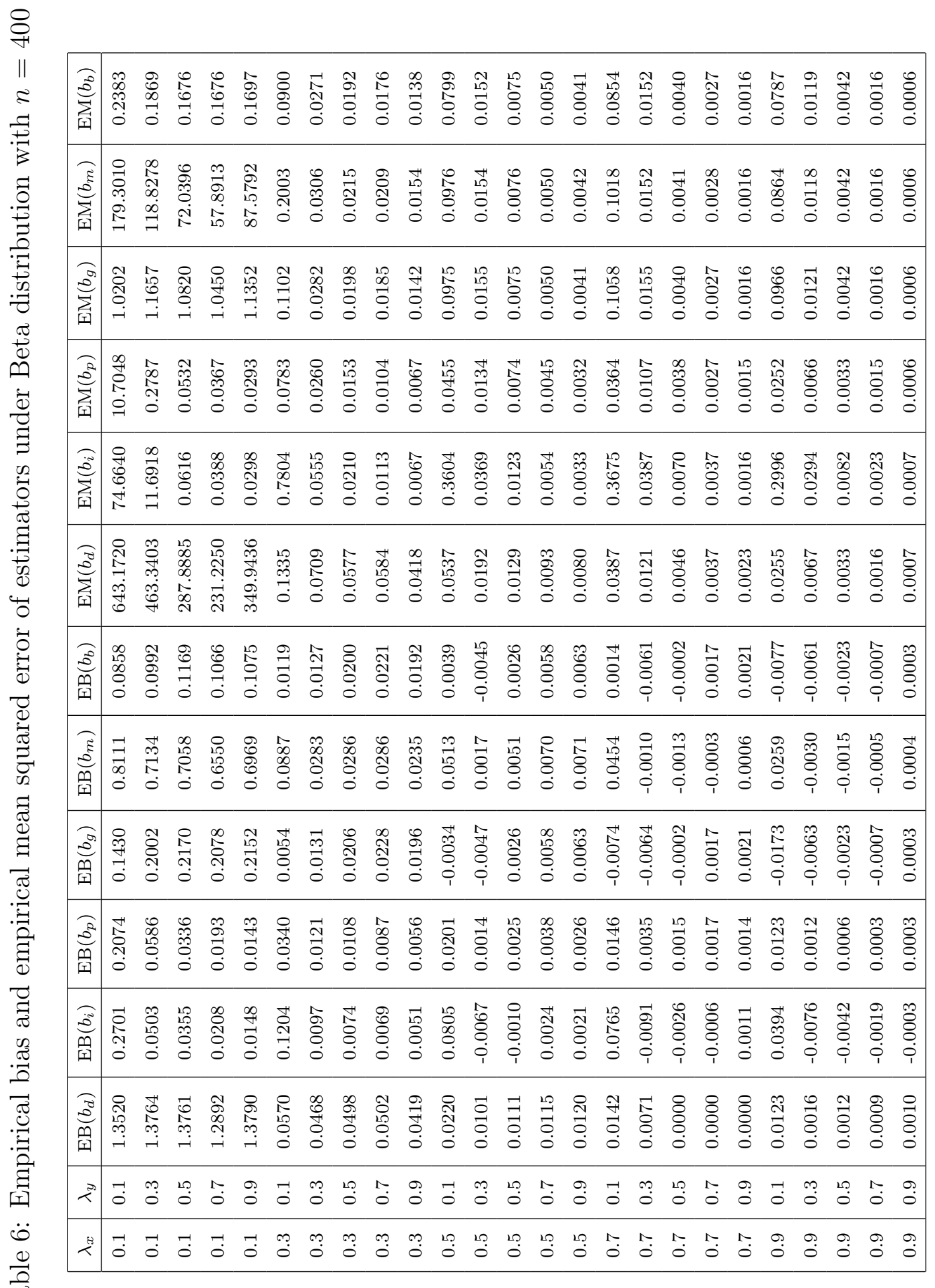




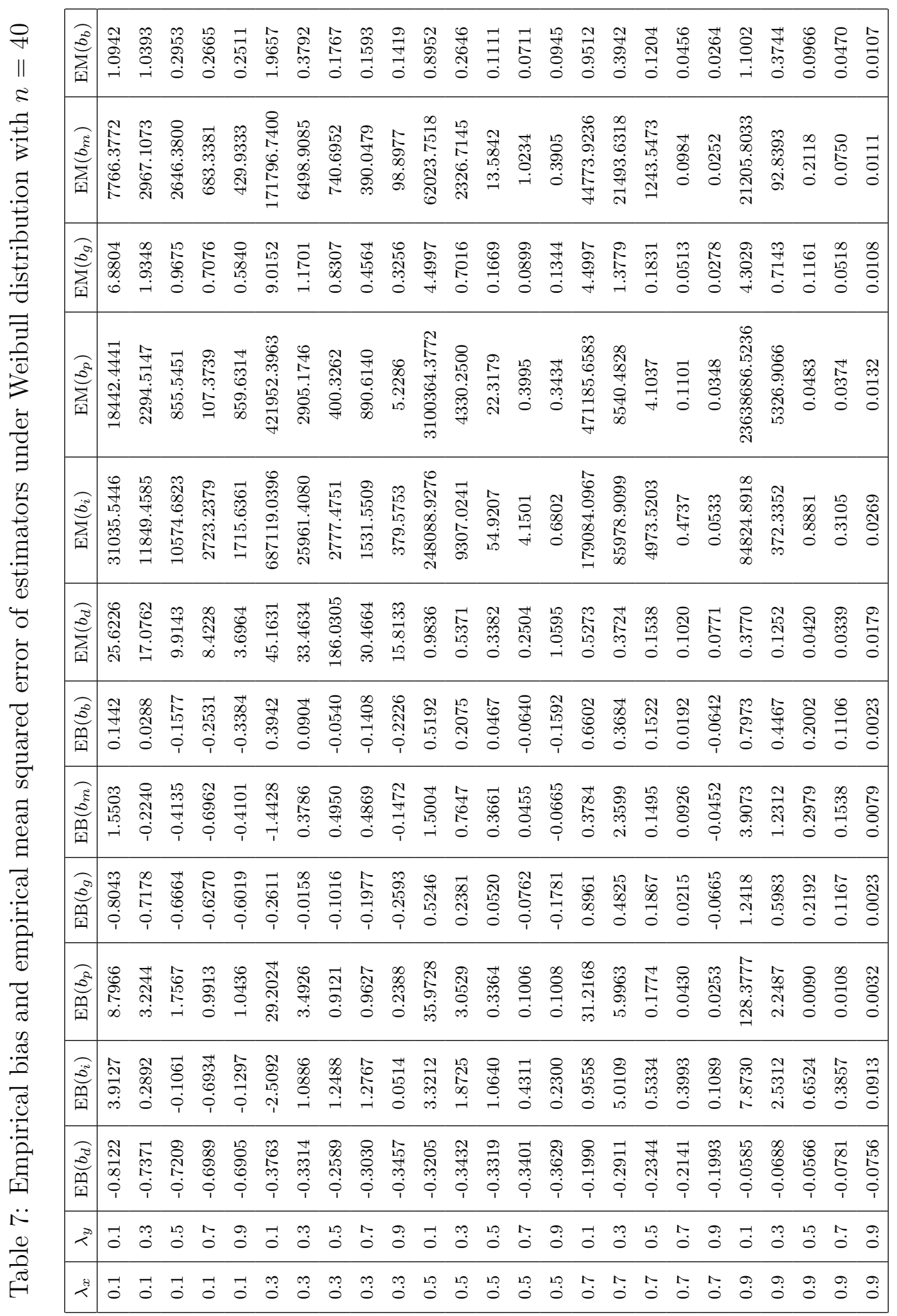




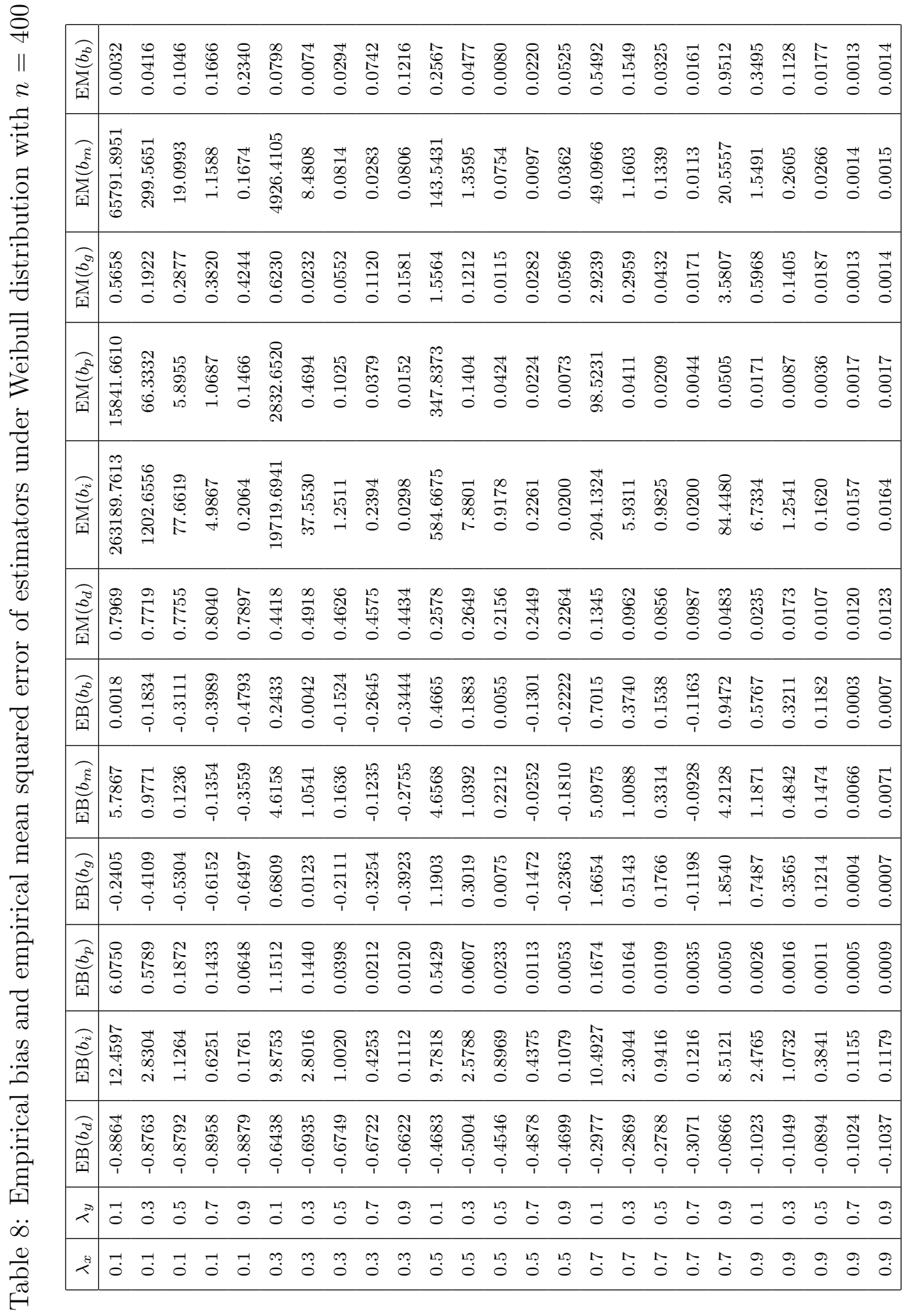

